\title{
La mise en œuvre de la définition et de la protection par les DIREN
}

Dominique Saumet

\section{(2) OpenEdition \\ Journals}

Édition électronique

URL : https://journals.openedition.org/rhcf/578

DOI : $10.4000 /$ rhcf. 578

\section{Éditeur}

Rails \& histoire

Édition imprimée

Date de publication : 1 novembre 2005

Pagination : 119-126

ISSN : 0996-9403

Référence électronique

Dominique Saumet, "La mise en œuvre de la définition et de la protection par les DIREN », Revue d'histoire des chemins de fer [En ligne], 32-33 | 2005, mis en ligne le 16 mai 2011, consulté le 22 avril 2022. URL : http://journals.openedition.org/rhcf/578 ; DOI : https://doi.org/10.4000/rhcf.578 


\section{Dominique SAUMET}

\section{La mise en œuvre de la définition et de la protection par les DIREN}

\section{Présentation de la DIREN}

En tant que services extérieurs du ministère de l'Écologie et du Développement durable (MEDD) les 22 directions régionales de l'Environnement (DIREN) sont normalement très imprégnées de la notion de patrimoine. Créées en 1991, elles sont les descendantes en droite ligne des services des délégués régionaux à l'Architecture et à l'Environnement, qui avaient pris la place en 1979 des conservations régionales des Bâtiments de France, services régionaux du ministère des Affaires culturelles qui géraient à l'époque à la fois les monuments historiques (loi de 1913), les sites inscrits et les sites classés au titre de la loi du 2 mai 1930. Les DIREN ont à gérer trois grands domaines patrimoniaux : la protection et la mise en valeur des sites et monuments naturels, les paysages, la protection de la nature (réserves naturelles et le réseau Natura 2000) et, enfin, la surveillance et le contrôle de l'eau et des milieux aquatiques tant du point de vue de la qualité que de celui de la quantité. Au-delà de ses prérogatives propres, elle participe également sous l'autorité du préfet à la concertation générale avec les autres administrations de l'État et les élus sur tous les projets d'aménagement du territoire (urbanisme : POS, PLU, SCOT ; infrastructures routières, ferroviaires, installations classées, éoliennes...) et sur lesquels elle a de nombreuses données environnementales à fournir aux conducteurs de projets publics ou privés. Elle est force de conseil et fait part de ses propres avis sur les nombreuses études d'impact qui accompagnent les enquêtes publiques, en veillant à faire respecter la réglementation environnementale.

\section{La ligne à grande vitesse et la DIREN Poitou-Charentes}

Le 13 janvier 1994, le Premier ministre a proposé d'engager les études du projet LGV-Sud-Europe-Atlantique alors appelé TGVAquitaine avec un débat public mené par le préfet de la région Aquitaine, coordinateur du projet de novembre 1995 à avril 1996. Par décision du 29 décembre 1999, le ministre de l'Équipement, des Transports et 
du Logement a demandé l'engagement des études de l'avant-projet sommaire (APS) entre Bordeaux-La Grave d'Ambarès et le nord d'Angoulême. Celles-ci furent lancées ainsi que toutes les concertations locales de 2001 à 2003.

C'est ainsi que la DIREN Poitou-Charentes, en liaison avec celle d'Aquitaine, a participé activement aux études conduites par Réseau ferré de France (RFF) sur les sections Bordeaux-Angoulême et Tours, d'une part lors du " porter à la connaissance » du maximum de données environnementales en sa possession et, d'autre part, en suivant l'élaboration des différentes études sous le rapport de la protection de la nature et des paysages. C'est ainsi que le dossier d'APS a été transmis en juillet 2003 au ministre des Transports quil'approuvait fin 2003 et donnait son autorisation à la conduite d'études nécessaires à la réalisation de l'enquête publique, dont de solides études d'impact de l'infrastructure projetée.

Lors du «porter à la connaissance » de toutes les données environnementales en sa possession, la DIREN a donc informé RFF sur les thèmes suivants :

a- les servitudes d'État opposables dont elle est la garante :

- les sites inscrits et les sites classés au titre de l'article L 341 du code de l'environnement (ex loi du 2 mai 1930);

- les réserves naturelles au titre du code de l'environnement ;

- les arrêtés de protection de biotopes.

b- Le domaine des inventaires :

- les zones naturelles d'intérêt écologique faunistique et floristique (ZNIEFF);

- les zones d'intérêt communautaire pour les oiseaux (ZICO), les espaces « directives oiseaux » et « directives habitats » concernant la politique du réseau des espaces Natura 2000, les arbres remarquables inventoriés dans les communes traversées par le fuseau des tracés étudiés ;

- les paysages sensibles répertoriés à l'atlas des paysages de PoitouCharentes;

- toutes les données utiles sur l'eau en matière de qualité et de quantité.

Dans une concertation très active et loyale, le service de la délégation régionale de RFF de Bordeaux, aidé de différents bureaux d'études spécialisés en environnement, a rencontré la DIREN à plusieurs reprises au fur et à mesure de l'avancement des études et en fonction des différents problèmes rencontrés sur le fuseau. Des études complémentaires sur certains milieux naturels ont même été réalisées à 
la demande de la DIREN pour mesurer les impacts liés à la réalisation de la voie qui peut jouer le rôle de barrage pour certaines espèces animales.

Faire passer une grande structure linéaire ex-nibilo, telle une autoroute ou une ligne de chemin de fer LGV avec ses caractéristiques propres, relève en 2005 d'un défi par rapport à la création des premières voies ferrées du XIx ${ }^{e}$ siècle, qui épousaient davantage le terrain. Même si celles-ci suscitaient de brutales réactions de la part des populations locales qui avaient peur qu'avec la vapeur leurs vaches deviennent folles avant l'heure, même si des élus prenaient fortement parti pour ou contre la venue du rail et des gares, même si de nombreuses archives témoignent de l'âpreté des rapports entre les préfets de la III ${ }^{e}$ République poussant au développement du réseau ferré et certains grands élus locaux opposés à l'arrivée du rail, il y avait pour les ingénieurs de l'époque beaucoup moins de problèmes environnementaux à surmonter qu'aujourd'hui. En revanche, ils avaient à relever d'autres défis, en particulier celui de faire des prouesses dans la conception et la construction d'ouvrages d'art en maçonnerie classique, tel le viaduc de Morlaix, ou en construction d'avant-garde avec le fer puis l'acier, tels les ouvrages de Gustave Eiffel, lors des accidents de terrain, sans oublier les nombreux tunnels.

De nos jours, les aspects juridiques se sont multipliés et renforcés avec les réglementations nouvelles et nombreuses des codes de l'environnement, de l'urbanisme... Par ailleurs, le territoire s'est développé partout : autoroutes, routes, déviations, lignes électriques 400/200/ $90 \mathrm{~kW}$, zones industrielles, commerciales à n'en plus finir autour des villes, et les nombreuses zones mitées en périphérie des centres urbains, sans oublier ici et là les pelouses sèches à orchidées à éviter, les derniers restes de zones humides qu'il ne faut pas remblayer... Tout cela ajouté ne facilite pas le passage d'un nouveau projet de voie ferrée LGV qui doit slalomer entre toutes ces contraintes.

Au-delà de l'administration, ces concepts de la protection de la nature et des paysages ont été repris par le monde associatif des anciennes sociétés savantes devenues associations départementales de protection de la nature et de l'environnement, qui peuvent également apporter leur contribution de spécialistes pour certains milieux particulièrement fragiles ou ayant une connotation écologique forte dans notre région (boisements, pelouses sèches à orchidées déjà citées, zones humides...).

La DIREN, avec ses compétences et ses missions dans les domaines de l'eau, la nature, le paysage et le cadre de vie, conseille et aide RFF à trouver les solutions pour minimiser au mieux les impacts négatifs et, en particulier, celui de la fragmentation des territoires. Pour 
des raisons de sécurité les LGV, comme les autoroutes, sont encloses par des grillages faisant obstruction au passage du gros gibier. De fait elles créent ainsi des barrières génétiques que les différents passages à gibier et autres crapauducs ne parviennent pas toujours à réduire.

La DIREN souhaite aussi que RFF s'engage à réaliser des ouvrages esthétiques, bien profilés et architecturés pour enjamber au mieux les faibles changements de relief de la région que sont les vallées, en particulier celles de la Charente et de la Vienne, en évitant au maximum le recours systématique aux remblais en fond de vallée qui font barrage à l'écoulement des eaux de crues en zone inondable et constituent un écran à la transparence du paysage dans la vallée. Le vieux réseau ferré de France était remarquable par la qualité esthétique du moindre ouvrage d'art passant au dessus d'un ruisseau, inutile ici de rappeler la qualité, la solidité et la beauté de ses ponts et de ses viaducs. Le grand enjeu paysager est que cette nouvelle infrastructure puisse recomposer l'espace là où elle passe sans en amoindrir la qualité. C'est à travers l'étude d'impact, outil très performant, que RFF doit nous en faire la démonstration.

La LGV est une infrastructure majeure qui, en elle-même, est à la fois un élément structurant du paysage et, de toute évidence, source de paysage nouveau. Elle est également un moyen pour découvrir le paysage et de nouveaux paysages différents de ceux de la route nationale 10, de l'autoroute A 10 ou de la vieille ligne SNCF Paris-Bordeaux qui, de Chatellerault à Angoulême, serpente dans les vallées successives du Clain et de la Charente.

En prenant en compte la dimension touristique du projet, l'accent a été mis également sur des études paysagères spécifiques et complémentaires sur le paysage vu du train. Le train a été de tous temps le moyen de découverte de paysages nouveaux. Dans l'immense collection des affiches publicitaires, les sites et les paysages d'exception de Londres à Istamboul en passant par le Mont-Saint-Michel et Menton ont servi de support à plus de cent ans de promotion du chemin de fer en Europe. Ainsi, jeune lycéen, je découvrais de 1960 jusqu’à 1969, comme des milliers d'autres lycéens avant moi, ces splendides illustrations du chemin de fer qui ornaient les couloirs, les réfectoires et les dortoirs du lycée Descartes à Tours et qui excitaient davantage ma forte passion pour le rail, les locomotives, les gares de triage et les voyages debout dans le couloir à la fenêtre pour voir le paysage défiler à 120 - $140 \mathrm{~km} / \mathrm{h}$ et sentir l'air des champs mélangé aux effluves exhalés de la voie ferrée.

Avant l'arrivée des trains Corail, première régression avant les TGV en matière de contact avec le paysage si visible des grandes fenêtres des voitures, chaque voyageur avait au-dessus de sa tête, sous les filets 
des bagages, ou bien face à lui, de splendides photographies en noir et blanc montrant les plus beaux sites et paysages de la France.

Alors aujourd'hui comment s'analyse la perception du paysage à $300 \mathrm{~km} / \mathrm{h}$ ? D'abord le regarde-t-on encore aujourd'hui, comme naguère collé à la vitre du couloir ? Sans doute beaucoup moins qu'avant, mais la réponse demeure encore positive malgré les apparences de préoccupation des voyageurs occupés à travailler sur leur ordinateur portable ou à lire. Car le train permet d'admirer de façon beaucoup plus détendue qu'en voiture le paysage qui apparaît à la fenêtre. Qu'observe-t-on dans ce qui défile à toute allure ? Quelle est la profondeur de champ visible et surtout lisible ? Le deuxième, le troisième ou l'arrière-plan plus statique ? Et puis il arrive au train de ralentir pour entrer dans les zones urbaines et leurs gares.

Dans ce cas, quels seraient alors les éléments négatifs de paysage à améliorer vus du train, et les éléments positifs à mettre en avant, de jour comme de nuit, avec une scénographie de certains monuments ou sites à mettre en valeur, ainsi que les lieux emblématiques traversés, point de repère du voyageur au passage de certaines villes ? C'est dire les progrès qui ont été faits dans ce domaine et l'importance donnée à ce volet paysager qui s'étire sur des centaines de kilomètres.

\section{Autres domaines}

Un autre domaine qui préoccupe la DIREN par rapport à l'environnement ferroviaire est le devenir des anciennes voies ferrées, décrétées non rentables et souvent abandonnées à leur triste sort. Certaines encore reliées à des silos servent de liaison pour le transport des céréales, autant de milliers de camions en moins sur les routes. Au-delà du silo les voies ont été déclassées, la proie d'une végétation sauvage, ou transformées en chemin de randonnée, ou en parcours « vélorail » ou, pire encore, vendues. Mais si demain une autre économie dans un nouveau contexte énergétique nous forçait à revoir la copie du comportement individualiste du tout automobile et nous obligeait à penser davantage transport en commun, ce qui arrivera un jour? Ne serait-on pas heureux de redécouvrir des voies quasiment prêtes à l'emploi ? C'est pourquoi il est important de sauvegarder le plus possible ce patrimoine ferré qui irriguait naguère si bien tout le territoire. Il semblerait ainsi que l'on soit davantage dans l'esprit du développement durable dont on parle tant depuis peu... 


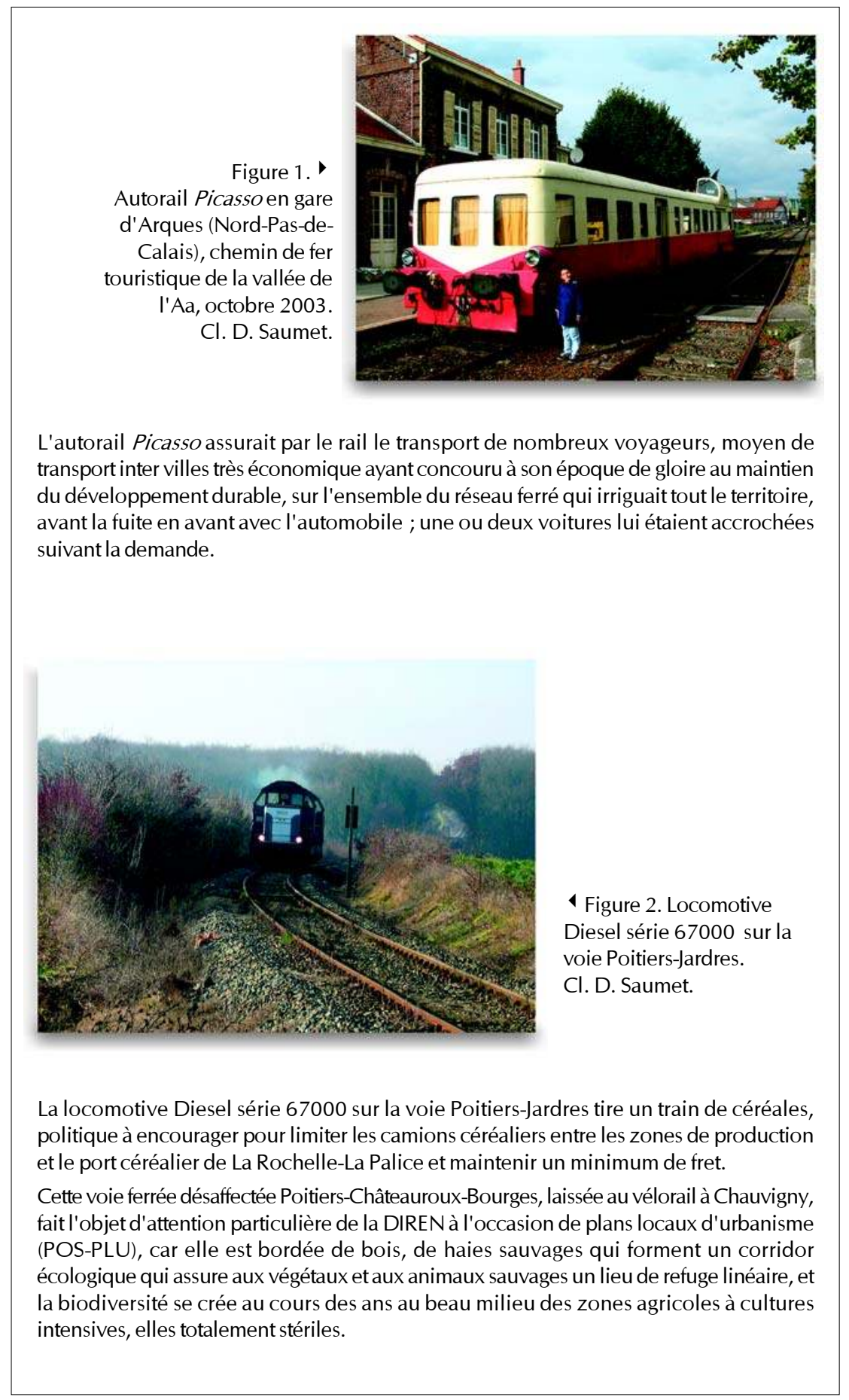




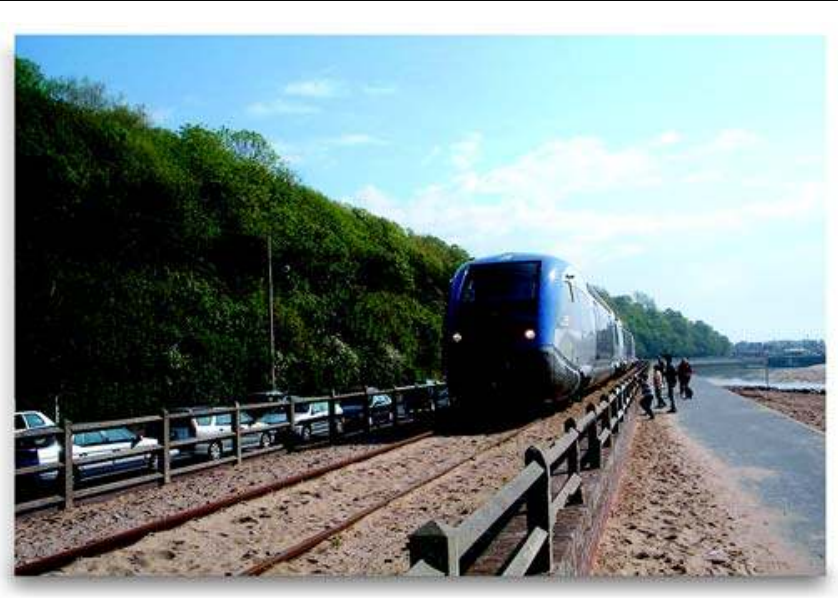

4 Figure 3. Le TER passe sur la plage à Houlgate-surMer, mai 2002. Cl. D. Saumet.

Sur la ligne côtière normande : ligne Cabourg-Dives-sur-Mer-Deauville puis LisieuxParis, le voyageur sort du bocage normand et arrive les roues dans l'eau de mer de la Côte Fleurie. La région Basse-Normandie a mis en place une politique ferroviaire de desserte importante liant ces principaux centre urbains : Cherbourg, Saint-Lô, Bayeux, Caen, Lisieux.

Figure 4. Le pont de Busseausur-Creuse à Ahun, ouvrage d'art de l'ingénieur Lloyd, avril 2004.

Cl. D. Saumet.

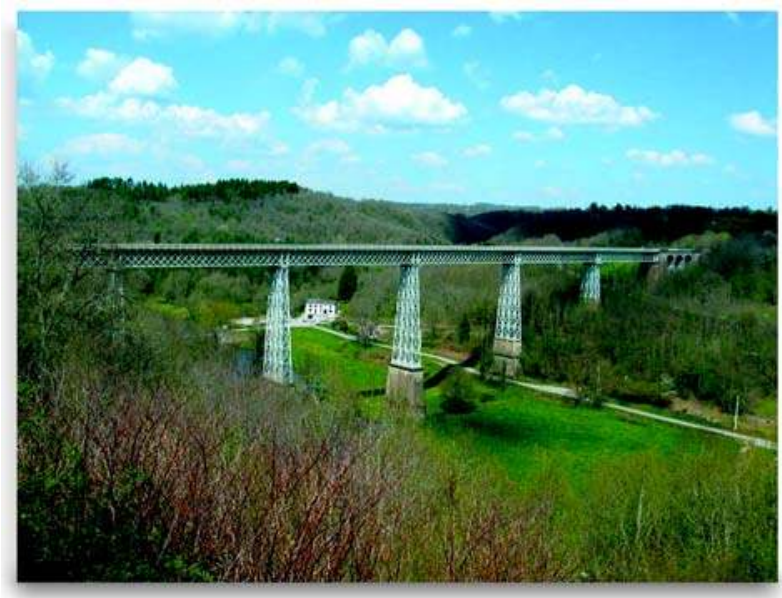

Le pont de Bussereau-sur-Creuse a été construit en moins d'un an (de 1863 à 1864) sur la ligne SNCF Bordeaux-Genève pour réaliser la ligne Limoges-Monluçon de la Compagnie du PO. 
Dans un autre domaine, à l'occasion des réunions sur les plans d'occupation des sols ou les plans locaux d'urbanisme, la DIREN attire également l'attention des élus sur les dépendances vertes qui longent les anciennes voies ferrées en service ou non et qui sont devenues des corridors biologiques de toute première importance, de véritables refuges linéaires de la faune et de la flore. Ce sont des oasis écologiques au beau milieu des plaines totalement stérilisées par les intrants liés à la monoculture intensive du blé, du maïs ou du colza... Tout comme naguère la vieille ligne de Sceaux, au beau milieu de la banlieue sud parisienne, était à la fois dans ses remblais et déblais le lieu de refuge des insectes, des mollusques, des plantes sauvages, et aussi celui des professeurs du laboratoire de biologie évolutive et d'écologie de l'université Paris-VI. Ces grands biologistes que nous vénérions nous faisaient découvrir, à notre grande stupéfaction, la richesse biologique de ces milieux linéaires et étroits en y faisant une partie de nos travaux pratiques in vivo... Pour porter le minimum d'atteinte à ces milieux et surtout diminuer au maximum la pollution des réseaux hydrographiques proches des voies ferrées ou passant dessous, des efforts ne seraient ils à faire en France (comme le fait déjà depuis longtemps la Deutsche Bundesbahn) pour ne pas surtraiter le domaine ferré avec des produits désherbants extrêmement dangereux ? Les produits particulièrement efficaces employés naguère par la SNCF étaient d'ailleurs bien connus du monde agricole qui les convoitait jalousement.

Ainsi, cette merveilleuse infrastructure qu'est le rail, son matériel roulant et ses cheminots, rassemble depuis plus de 150 ans un patrimoine d'une richesse inouie, ayant ses ramifications innombrables dans la littérature, la peinture, la photographie, le cinéma, la sociologie, l'actualité. A $300 \mathrm{~km} / \mathrm{h}$ elle nous fait toujours découvrir, plus vite certes, les magnifiques séquences paysagères qui s'enchainent entre Paris et Lyon : du Morvan et des monts du Mâconais on dévale rapidement dans les Dombes. Demain, on planera de la Beauce en sautant la Loire, la Vienne, puis la Charente pour plonger dans la Gironde. Les lignes classiques ont toujours le droit d'exister et de servir, elles possèdent toute leur noblesse et leur agrément et rendent un service public loin d'être négligeable ; elles seront demain un plaisir nouveau et retrouvé de prendre le train tranquillement, tout comme certaines lignes touristiques avec train à vapeur connaissent un immense succès pour aller découvrir des sites, des paysages merveilleux avec ce petit plaisir de nostalgie bien ancrée dans chacun de nous. Enfin, tout comme les dépendances vertes des routes nationales et des autoroutes, un fil patrimonial biologique de verdure ferroviaire existe, où se retrouvent et s'adaptent une faune et une flore en parfaite harmonie avec le passage des trains et qui relie aussi nos cités. 